\title{
Coupling Dichloroacetate Treatment with Curcumin Significantly Enhances Anticancer Potential
}

\author{
PING-CHUAN KAN ${ }^{1}$, YU-JIA CHANG ${ }^{2,3}$, CHIN-SUNG CHIEN ${ }^{4}$, CHEN-YING SU ${ }^{5}$ and HSU-WEI FANG ${ }^{5,6}$ \\ ${ }^{1}$ Graduate Institute of Biochemical and Biomedical Engineering, \\ National Taipei University of Technology, Taipei, Taiwan, R.O.C.; \\ ${ }^{2}$ Division of General Surgery, Department of Surgery, Taipei Medical University Hospital, Taipei, Taiwan, R.O.C.; \\ ${ }^{3}$ Graduate Institute of Clinical Medicine, College of Medicine, Taipei Medical University, Taipei, Taiwan, R.O.C.; \\ ${ }^{4}$ Graduate Institute of Clinical Medicine, College of Medicine, National Taiwan University, Taipei, Taiwan, R.O.C.; \\ ${ }^{5}$ Department of Chemical Engineering and Biotechnology, \\ National Taipei University of Technology, Taipei, Taiwan, R.O.C.; \\ ${ }^{6}$ Institute of Biomedical Engineering and Nanomedicine Research, \\ National Health Research Institutes (NHRI), Miaoli, Taiwan, R.O.C.
}

\begin{abstract}
Background/Aim: Dichloroacetate (DCA) and curcumin have been shown to be potent drug candidates in cancer therapy. Our study aimed to investigate the combined effects of DCA and essential oil-blended curcumin (ECUR) using the hepatoma Huh-7 cell model. Materials and Methods: Muse ${ }^{T M}$ Cell Cycle assay, Muse ${ }^{T M}$ Annexin V \& Dead Cell assay, Muse ${ }^{T M}$ Oxidative Stress assay, and western blot analysis were applied to explore the underlying mechanisms. Results: DCA combined with ECUR dramatically augmented inhibition of cell survival and enhanced apoptotic induction. The enhanced apoptosis was accompanied by mitochondria-dependent apoptotic signaling activation and corroborated with significant cellular morphological alternations. Conclusion: Apoptosis was the major event contributing to the synergistically boosted antiproliferative effect. Coupling DCA treatment with curcumin may rationally be expected to lower the DCA dose needed and relatively reduce accompanying toxicity and oxidative damage while enhancing anticancer potential. This novel 'add-on' approach is, thus, of enormous value to the current DCA therapy.
\end{abstract}

Correspondence to: Hsu-Wei Fang, Department of Chemical Engineering and Biotechnology, National Taipei University of Technology, 1, Sec. 3, Zhongxiao E. Rd., Taipei 10608, Taiwan, R.O.C. E-mail: crntut@gmail.com or Ping-Chuan Kan, Graduate Institute of Biochemical and Biomedical Engineering, National Taipei University of Technology, 1, Sec. 3, Zhongxiao E. Rd., Taipei 10608, Taiwan, R.O.C. E-mail: biontut@gmail.com

Key Words: Combination therapy, dichloroacetate, curcumin.
Hepatocellular carcinoma (HCC) represents about $90 \%$ of primary liver cancers and ranks as the second cause of cancer-associated death globally (1). Despite increased understanding of hepatocarcinogenesis, limited treatment options have been established to improve survival for patients suffering from unresectable advanced HCC (2). Dichloroacetate (DCA), a synthetic pyruvate dehydrogenase kinase inhibitor, has been investigated as a novel metabolic therapeutic for various types of cancer (3-5). Akbar Khan et al. reported that observational data, collected from more than 300 patients with advanced-stage cancer, revealed measurable benefits from DCA therapy in $60-70 \%$ of cases (6). DCA targets cancer energy metabolism by inhibiting pyruvate dehydrogenase kinase, increasing pyruvate flux into the mitochondria, promoting glucose oxidative phosphorylation, and thereby reverses the glycolytic phenotype and overcomes the Warburg effect (5). This shift in metabolism allows for increased reactive generation of oxygen species (ROS) and cytochrome $c$ translocation from the mitochondria to cytoplasm, subsequently inducing cancer cell apoptosis through caspase cascade activation (3).

Curcumin, exhibiting multiple anticancer mechanisms, is fit to be a front-runner among anticancer agents as is a pharmacologically safe compound which can play a part in both prevention and treatment of cancer (7). Among multiple anticancer mechanisms, inhibition of nuclear factor-kappa B (NF-kB) seems particularly important. A previous study has delineated the mechanisms involved in curcumin-mediated suppression of NF-kB expression, which mediates proliferation, invasion, angiogenesis and metastasis in cancer (8). However, although in vitro and in vivo studies have shown anticancer activities of curcumin for virtually all types of human cancer, its poor 
bioavailability in the human body has severely limited its application to these diseases (9). Reconstituting curcumin with turmeric essential oil has been found to increase its bioavailability by around 7-fold compared to curcumin alone (9). Therefore, essential oil-blended curcumin (ECUR) was employed in this study.

Combination therapy appears to be a promising strategy for patients with $\mathrm{HCC}$, due to the fact that it can improve efficacy and also alleviate the adverse effects of drugs (10). We assumed that coupling DCA treatment with ECUR may enhance the anticancer potential against HCC. To test this hypothesis, this study investigated the combined effect of DCA and ECUR using human HCC Huh-7 cell model and tried to explore the underlying mechanisms.

\section{Materials and Methods}

Materials. DCA and sorafenib were purchased from Sigma-Aldrich Chemical Company (St. Louis, MO, USA). ECUR (commercial name: BIOCURCUMAX) was obtained from Arjuna Natural Ltd (Kerala, India). All agents were dissolved in dimethyl sulfoxide (DMSO) and diluted to appropriate concentrations with culture medium. The final DMSO concentration was $0.1 \%(\mathrm{~V} / \mathrm{V})$. DMSO was added to cultures at $0.1 \%(\mathrm{~V} / \mathrm{V})$ as a vehicle control.

Cell culture. Huh-7 cells (obtained from the Health Science Research Resources Bank, Osaka, Japan) were maintained in Dulbecco's modified Eagle's medium (Sigma-Aldrich, Saint Louis, MO, USA) supplemented with $10 \%$ fetal calf serum. Cells were cultured in an incubator with humidified air containing $5 \% \mathrm{CO}_{2}$ at $37^{\circ} \mathrm{C}$.

Cell survival assay. Cell viability assay was performed using Cell Counting Kit-8 (CCK-8) (Dojindo, Kumamoto, Japan). Briefly, cells $\left(1 \times 10^{3}\right)$ were seeded in 96 -well plates and treated with DCA $(0,15$, $20,25,30$ and $50 \mathrm{mM})$, alone or in combination with ECUR $(30 \mu \mathrm{M})$ for $48 \mathrm{~h}$. Increasing concentration of sorafenib $(2,4,6,8,10$ and 12 $\mu \mathrm{M})$ was used as positive control. The inhibitory effects of treatment with DCA $(40 \mathrm{mM}), E C U R(40 \mu \mathrm{M})$, and their combination for 16 and $24 \mathrm{~h}$ were further investigated. All experiments were performed in triplicate. Cell survival was determined using the OD value at 450 $\mathrm{nm}$ according to the manufacturer's instructions.

Cell-cycle distribution assay. Huh-7 cells $\left(1 \times 10^{6}\right)$ were seeded and treated with vehicle, DCA $(40 \mathrm{mM})$, ECUR $(40 \mu \mathrm{M})$, or their combination for 16 and $24 \mathrm{~h}$. The treated cells were subjected to cell-cycle assay using Muse ${ }^{\mathrm{TM}}$ Cell Cycle kit (Millipore, Billerica, MA, USA) according to the manufacturer's instructions. The relative percentages of cells in each phase were analyzed using Muse $^{\mathrm{TM}}$ Cell Analyzer (Millipore).

Cell apoptotic assay and cellular morphological examination. Huh7 cells were treated with vehicle, DCA $(40 \mathrm{mM})$, ECUR $(40 \mu \mathrm{M})$ or their combination for 16 and $24 \mathrm{~h}$. The treated cells were subjected to cell apoptotic assay using multifunctional Muse ${ }^{\mathrm{TM}}$ Annexin V and Dead Cell kit (Millipore) according to the manufacturer's instructions. The relative percentages of early apoptotic, late apoptotic, necrotic and visible cells were analyzed using Muse $^{\mathrm{TM}}$ Cell Analyzer (Millipore, Billerica, MA, USA).
Apoptosis was further confirmed by examining cell morphology changes using an inverted Motic AE31E microscope (Stellar Scientific, Baltimore, MD, USA).

Protein extraction and western blot analysis. The treated cells were harvested and lysed using ice-cold RIPA buffer (Cell Signaling, Danvers, MA, USA) containing protease inhibitors. Protein concentrations were determined using BCA assay (VisualProtein, Taipei, Taiwan, ROC). All protein samples were prepared in reducing sample buffer and separated on $15 \%$ sodium dodecyl sulfatepolyacrylamide gels, and then electro-transferred to polyvinylidene fluoride membranes for immunoblotting. Target proteins were probed using antibodies against caspase-7 (Novus Biologicals, Littleton, CO, USA), caspase-8 (Cell Signaling), caspase-9 (Cell Signaling), and Bcell lymphoma-extra large (BCL-xL) (Cell Signaling). $\beta$-Actin (Abcam, Cambridge, MA, USA) was used as an internal control. Horseradish peroxidase-conjugated secondary antibodies (Cell Signaling) were used to recognize the corresponding primary antibodies.

Measurement of intracellular ROS. Huh-7 cells were treated with vehicle, DCA $(40 \mathrm{mM})$, ECUR $(40 \mu \mathrm{M})$ or their combination for 8,16 and $24 \mathrm{~h}$. The treated cells were subjected to ROS assay using Muse ${ }^{\mathrm{TM}}$ Oxidative Stress Kit (EMD Millipore, Billerica, MA, USA) according to the manufacturer's instruction. The relative percentages of $\mathrm{ROS}^{-}$and $\mathrm{ROS}^{+}$cells were analyzed using Muse ${ }^{\mathrm{TM}}$ Cell Analyzer (Millipore).

Coefficient of drug interaction. The coefficient of drug interaction (CDI) was used to analyze the effects of drug combination $(11,12)$, and derived as follows: $\mathrm{CDI}=A B /(A \times B)$, where $A B$ is the absorbance ratio of the combination to control; $\mathrm{A}$ and $\mathrm{B}$ are the ratios obtained after treatment with single agents $\mathrm{A}$ and $\mathrm{B}$. Thus, CDI values $<1$, $=1$ and $>1$ indicate that drugs act synergistically, additively or antagonistically, respectively. CDI values of less than 0.7 indicate significant synergistic effects.

Statistical analysis. GraphPad Prism 6.01 (GraphPad Software, La Jolla, CA, USA) was used for graphical and statistical analysis. A one-way or two-way analysis of variance (ANOVA) with Tukey's multiple comparisons test was employed. Data were expressed as means \pm standard deviation (SD). A $p$-value of less than 0.05 was considered statistically significant.

\section{Results}

DCA and ECUR acted synergistically to suppress cell survival. Huh-7 cells were treated with increasing concentrations of $\operatorname{DCA}(0,15,20,25,30$ and $50 \mathrm{mM})$, or in combination with ECUR $(30 \mu \mathrm{M})$ for $48 \mathrm{~h}$. DCA treatment plus ECUR significantly reduced cell survival in a dosedependent manner compared to DCA treatment alone (Figure 1A). Increasing concentration of sorafenib (2, 4, 6, 8, 10 and $12 \mu \mathrm{M}$ ) was used as a positive control. The mean CDI values resulting from the 48-h combination treatments were all less than 1.0 and gradually reduced as the DCA concentration increased (Figure 1B). The inhibitory effects of treatment with DCA, ECUR and their combination for 16 and $24 \mathrm{~h}$ were further investigated. DCA treatment combined with ECUR significantly suppressed cell survival, compared with 

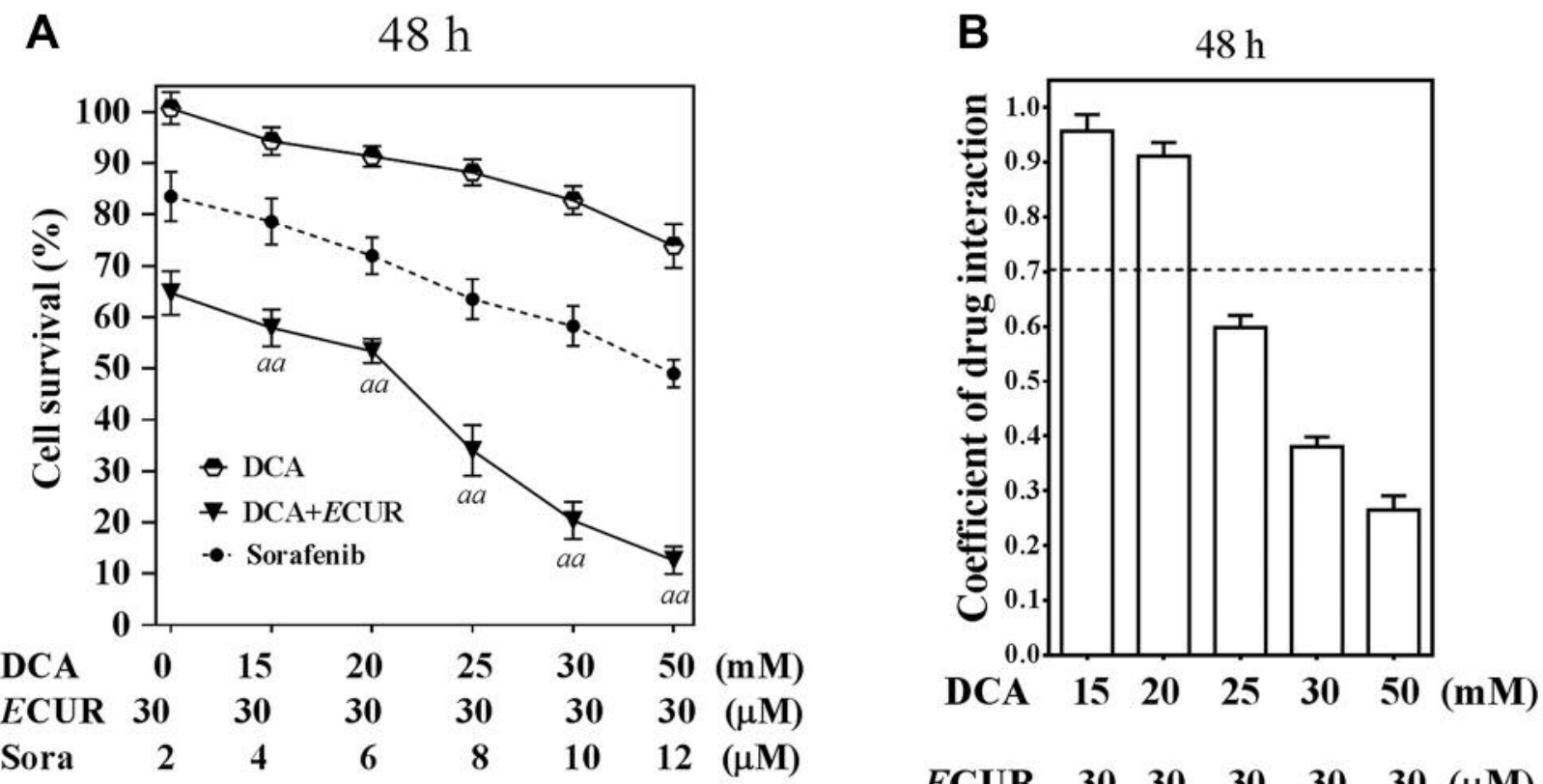

\section{$\begin{array}{lllllll}E C U R & 30 & 30 & 30 & 30 & 30 & (\mu \mathrm{M})\end{array}$}
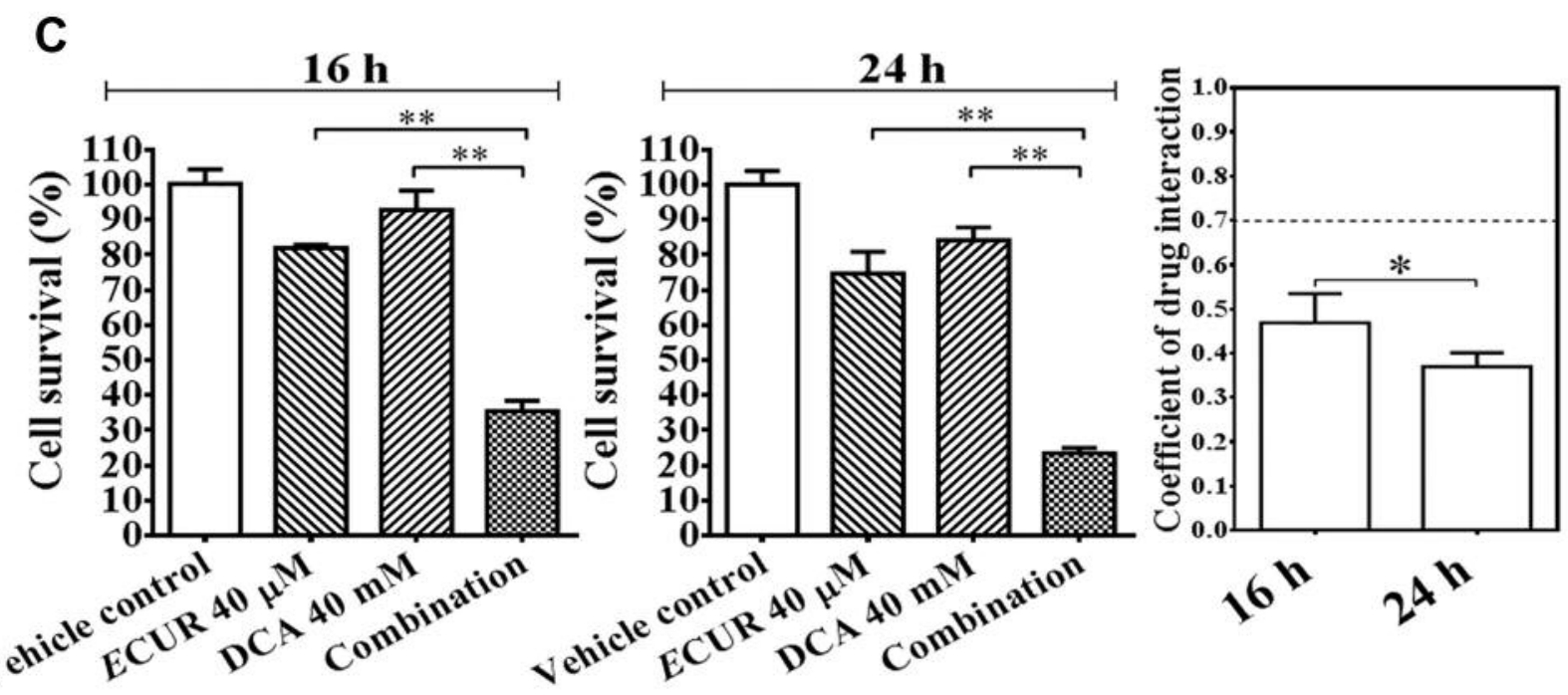

Figure 1. Cell survival analysis. Cell viability was measured by the CCK-8 assay. Dichloroacetate (DCA) in combination with essential oil-blended curcumin (ECUR) synergistically suppressed cell growth. A: Huh-7 cells were treated with increasing concentrations of DCA alone and in combination with ECUR for 48 h. Sorafenib (Sora) was used as a positive control. Significantly different at aa $p<0.01$ compared with DCA. C: Huh7 cells were further treated with vehicle, DCA, ECUR, or their combination for 16 and 24 hours. B, D: The coefficient of drug interaction (CDI) was used to analyze the effects of drug combination. $C D I>1$, antagonistic effect; $C D I=1$, additive effect; $C D I<1$, synergistic effect; CDI <0.7, significant synergistic effect. Data are presented as means $\pm S D(N=3)$. ANOVA followed by Tukey's multiple comparisons test, Significantly different at $* p<0.05$ and $* * p<0.01$.

both single treatments (Figure 1C). The mean CDI values resulting from the 16- and 24-hour combination treatments were calculated to be less than 0.7 (Figure 1D), indicating significant synergistic effect between DCA and ECUR.
DCA treatment alone and DCA treatment plus ECUR induced similar levels of $G_{0} / G_{1}$ cell cycle arrest. Following individual treatment of Huh-7 cells with DCA, ECUR or their combination for 16 and $24 \mathrm{~h}, \mathrm{G}_{0} / \mathrm{G}_{1}$ cell-cycle arrest 
was caused by DCA both alone and in combination with ECUR (Figure 2A and B). No obvious differences in the levels of arrest were observed between DCA treatment and the combination treatment (Figure 2B). Intriguingly, Wang et al. found that curcumin induced a slight $\mathrm{G}_{2} / \mathrm{M}$ arrest in Huh-7 cells (13), while our data from four independent experiments shown that ECUR did not provoke obvious $\mathrm{G}_{2} / \mathrm{M}$ phase arrest in Huh-7 cells.

The combination treatment promoted cell apoptosis and cellular morphological change. DCA treatment coupled with ECUR significantly increased the relative percentage of early apoptotic cells while reducing that of live cells compared with both single treatments (Figure 3A and B). No obvious necrosis was observed in any of the treated groups (Figure 3B). The increases in the relative percentage of total apoptosis were highly correlated with the increases in the relative percentage of inhibition of cell survival (Figure 3C). The occurrence of apoptosis was further confirmed by cellular morphological alternations. A small degree of nuclear fragmentation was shown following individual treatment with DCA and ECUR, and obvious nuclear breakup was observed after treatment with both agents (Figure 3D).

The combination treatment activated mitochondriadependent apoptotic pathway. Individual treatment of Huh7 cells with increasing concentrations of DCA (20, 30 and $40 \mathrm{mM})$ in combination with ECUR $(40 \mu \mathrm{M})$ for $24 \mathrm{~h}$ resulted in elevated expression of caspase-7 and-9 compared with the DMSO control, while the levels of caspase- 8 and BCL-xL were not obviously affected (Figure 4), indicating the activation of mitochondria-dependent apoptotic pathway.

DCA, ECUR and their combination induced intracellular ROS generation. To explore the potential role of ROS related to enhanced apoptosis, an ROS assay was conducted. After treatment of Huh-7 cells with DCA, ECUR or their combination for 8,16 and $24 \mathrm{~h}$, the relative percentage of ROS+ cells were determined. ROS+ cells arising from the combination treatment peaked at $8 \mathrm{~h}$, reaching a similar level as that caused by DCA, and then gradually decreased to almost the same level as that caused by ECUR (Figure 5).

\section{Discussion}

Evidence exists for the anticancer effects of DCA (6), but a high DCA dose is needed for significant therapeutic response (14). However, high-dose DCA treatment also produces detrimental side-effects involving dose-related peripheral neuropathy and encephalopathy (15), thereby impeding its application in cancer therapy $(14,15)$. This study revealed that DCA at doses only slightly effective in suppressing Huh7 cells dramatically enhanced inhibition of cell growth when applied with ECUR (Figure 1A). In addition, using a cytotoxic low dosage of $20 \mathrm{mM}$ DCA plus $30 \mu \mathrm{M}$ ECUR was more potently cytotoxic than $10 \mu \mathrm{M}$ of sorafenib (Figure 1A), which is currently the standard treatment for advanced HCC (16). These findings have great implication for potential clinical application as it suggests that coupling DCA treatment with ECUR may potentially allow the use of lower doses of DCA to achieve a given level of efficacy against HCC and thus, less toxic side-effects can also be expected.

Herein, the underlying mechanisms were explored. Cell-cycle arrest and cell apoptosis have been recognized as important mechanisms underlying inhibition of cell survival. As shown in Figure 2B, DCA both alone and in combination with ECUR induced $\mathrm{G}_{0} / \mathrm{G}_{1}$ cell cycle arrest in Huh-7 cells. However, no significant difference in the arrest levels were observed between both treatments. Subsequent apoptotic analysis further revealed that DCA treatment in combination with ECUR significantly enhanced cell apoptosis over either single treatment, and the increases in the relative percentage of total apoptosis were consistent with the increases in the relative percentage of cell survival inhibition (Figure 3C). Taken together, it suggests that apoptosis, rather than cell-cycle arrest, was the main event underlying enhanced inhibition of cell survival. In addition, DCA plus ECUR enhanced the induction of apoptosis through activating the mitochondria-dependent apoptotic pathway (Figure 4), suggesting the participation of BCL2 family members in apoptotic induction, as BCL2 family proteins mediate the mitochondria-dependent apoptotic event (17).

In HCC, expression of BCL2 is usually absent while BCL-xL is predominately expressed among the antiapoptotic BCL2 family members (18). The balance between pro-apoptotic and anti-apoptotic BCL2 family members determines the outcomes of $\mathrm{HCC}$ in terms of resistance or susceptibility to apoptotic induction (18). Indeed, the BCL$\mathrm{xL}$ protein was found to be highly activated in untreated Huh-7 cells (Figure 4), and the combination treatment did not show obvious effect on suppressing the overexpression of BCL-xL (Figure 4). Taken all together, this leads to the possibility that some proapoptotic BCL2 members may be activated which participate in the enhanced induction of apoptosis. Further study is needed to confirm this suggestion.

Targeting cancer therapy via ROS-based mechanisms has been proposed as a free radical therapeutic approach (19). However, excess ROS can also lead to chronic inflammation which could, in turn, mediate most chronic diseases, including cancer (20). Long-term exposure to DCA has been reported to induce hepatotoxic and hepatocarcinogenic precursor evidenced by significantly increased levels of oxidation-associated biomarkers in a murine model (21). Previous studies have shown that DCA (4) and curcumin (22) up-regulate the ROS level to enhance apoptotic induction in human hepatoma cells. Hence, the potential role of intracellular ROS related to enhanced apoptosis was investigated. As indicated in Figure 5B, the combination of 
A

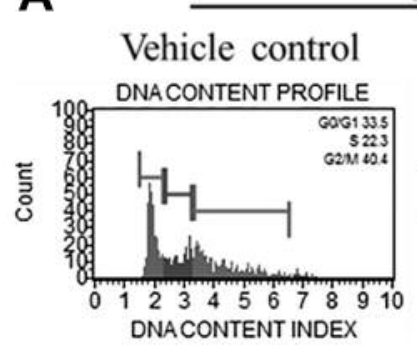

$16 \mathrm{~h}$
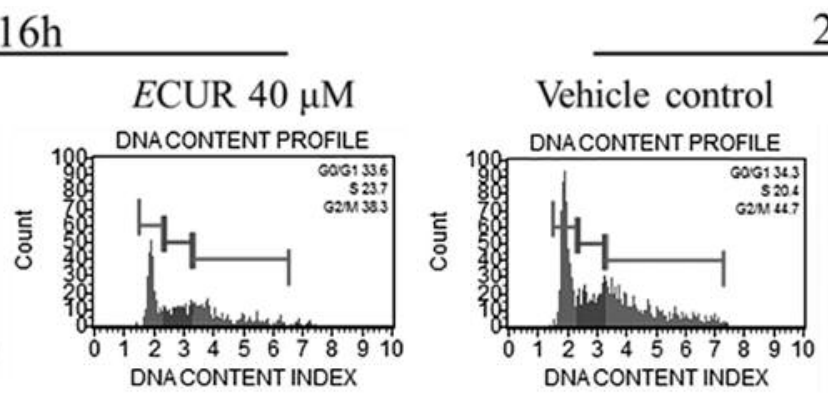

$24 \mathrm{~h}$

DCA $40 \mathrm{mM}$

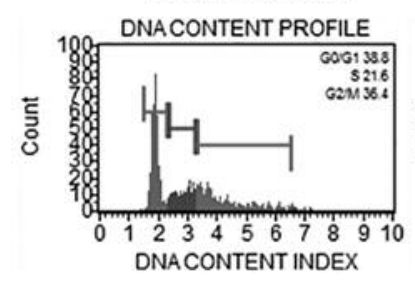

Combination
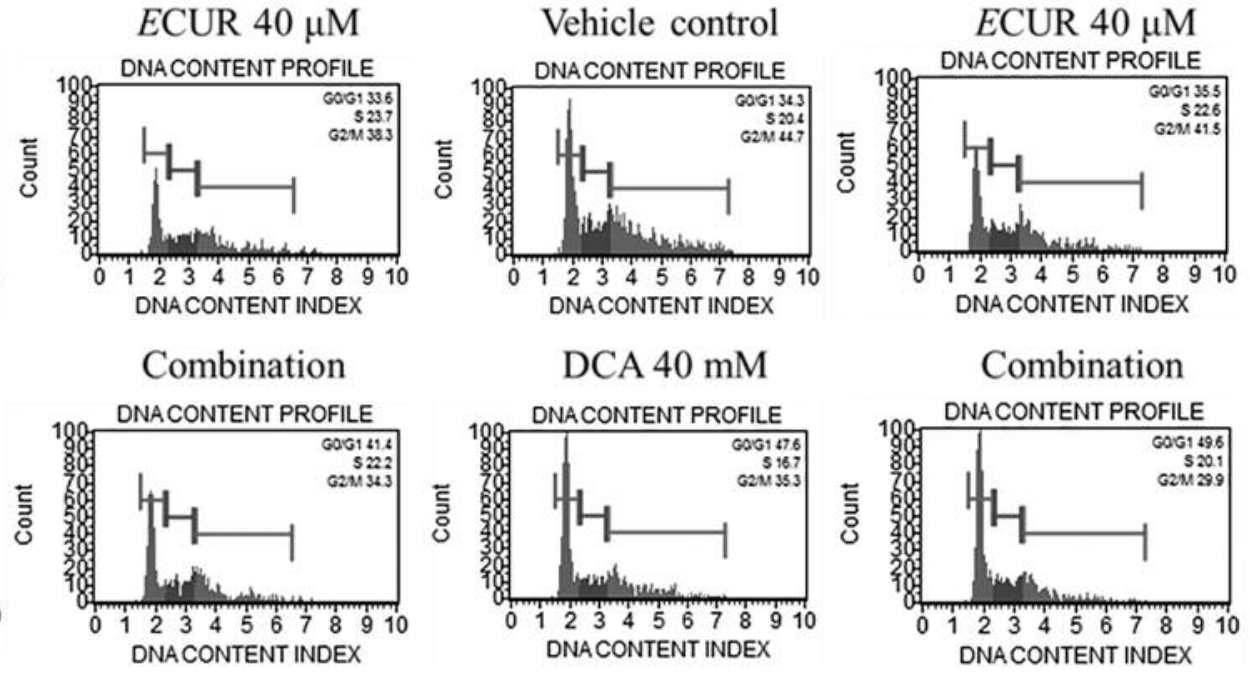

B

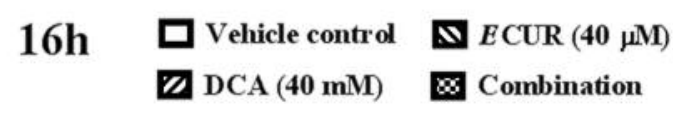

\section{4h $\square$ Vehicle control $\mathbb{E C U R}(40 \mu \mathrm{M})$ $\square \mathrm{DCA}(40 \mathrm{mM}) \quad \boldsymbol{B}$ Combination}
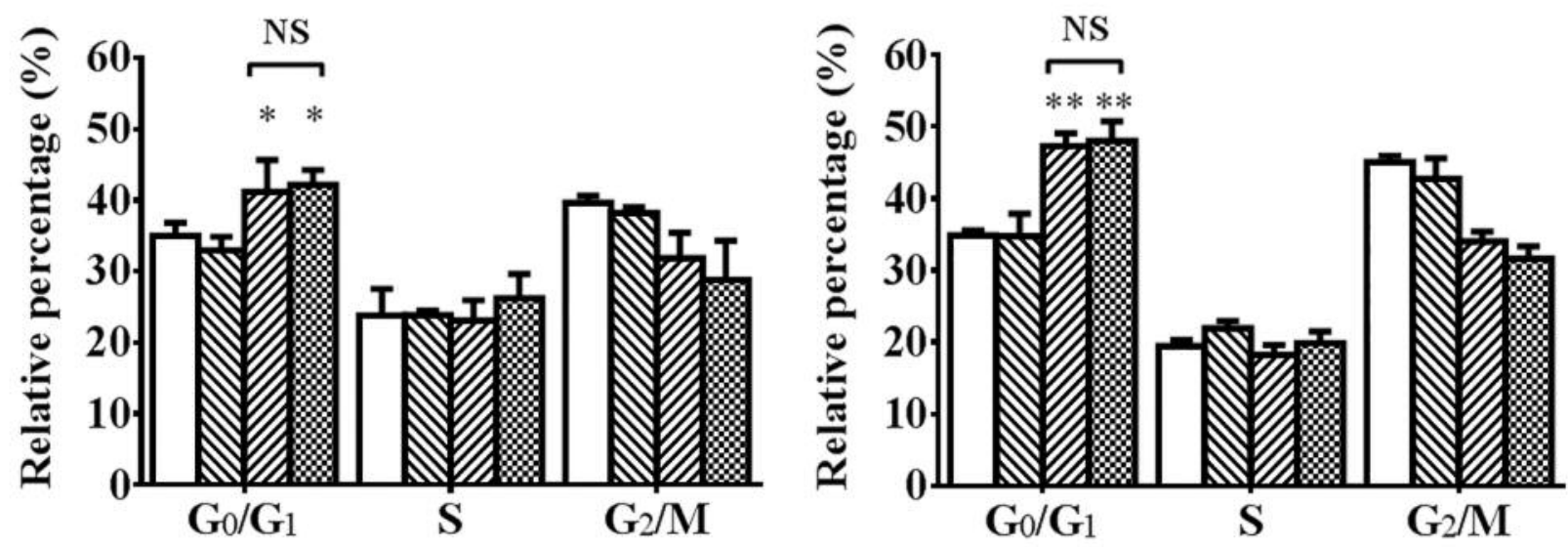

Figure 2. Quantitative cell-cycle distribution analysis. The cell-cycle distribution analysis shows the relative percentages of cells in each cell-cycle phase as measured by Muse ${ }^{T M}$ Cell Cycle assay. Huh-7 cells were treated with vehicle, dichloroacetate (DCA), essential oil-blended curcumin (ECUR), or their combination for 16 and 24 hours. A: The histogram is representative of four independent experiments. B: The quantitative graph shows that DCA treatment plus ECUR induced similar level of $G_{0} / G_{1}$ cell-cycle arrest as DCA treatment did. Data are expressed as means $\pm S D$ $(N=4)$. ANOVA followed by Tukey's multiple comparisons test, significantly different at $* p<0.05$ and $* * p<0.01$ compared with control.

the two potent pro-oxidants did not further increase ROS generation over single treatments, suggesting that intracellular ROS was not an effector triggering the apoptotic event. However, the reduction of ROS generation contributed by the addition of ECUR to DCA has great implication as it may reduce the potential risks of ROS-induced chronic lesions, particularly when long-term treatment is performed.
Overexpression of NF-kB is associated with poor prognosis in a variety of human cancer types (23), while it can be attenuated by curcumin (8). The Warburg effect, a common hallmark of cancer (24), is closely connected with proliferation, metastasis, angiogenesis, drug resistance and other aggressive behaviors of cancer cells, while it was effectively reversed by DCA $(5,25)$. Hence, coupling DCA 

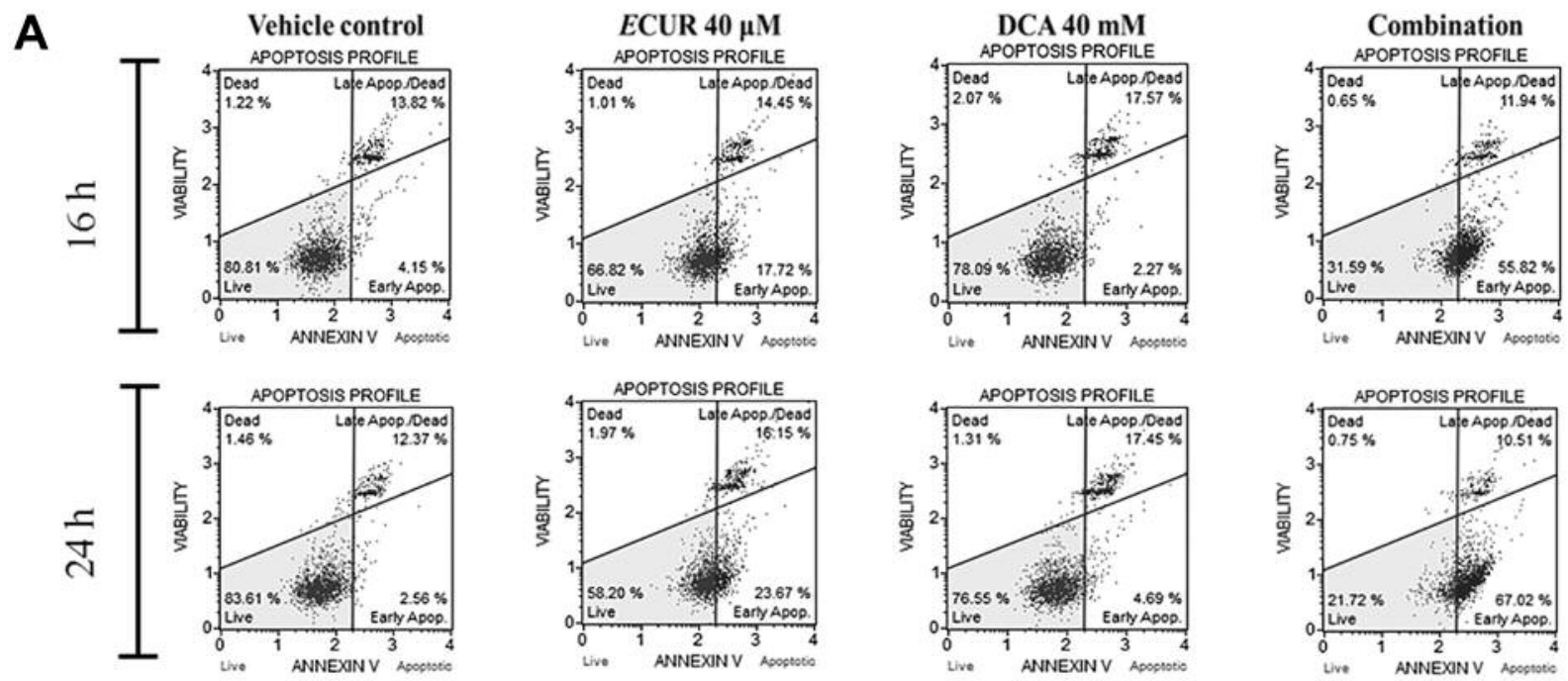

B

C

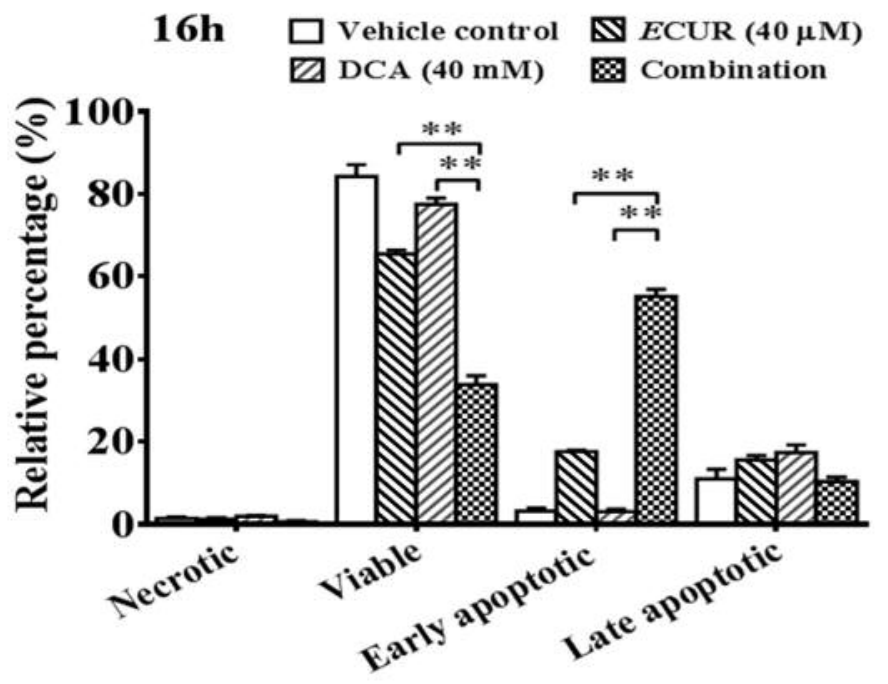

16h $⿴ 囗 十$ Total apoptosis (\%) Cell survival inhibition (\%)

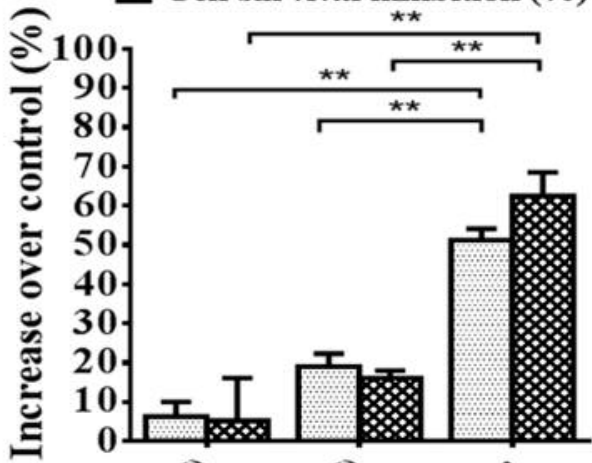

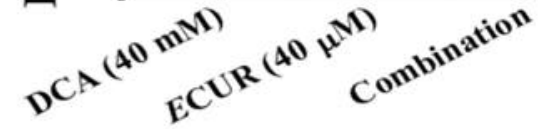
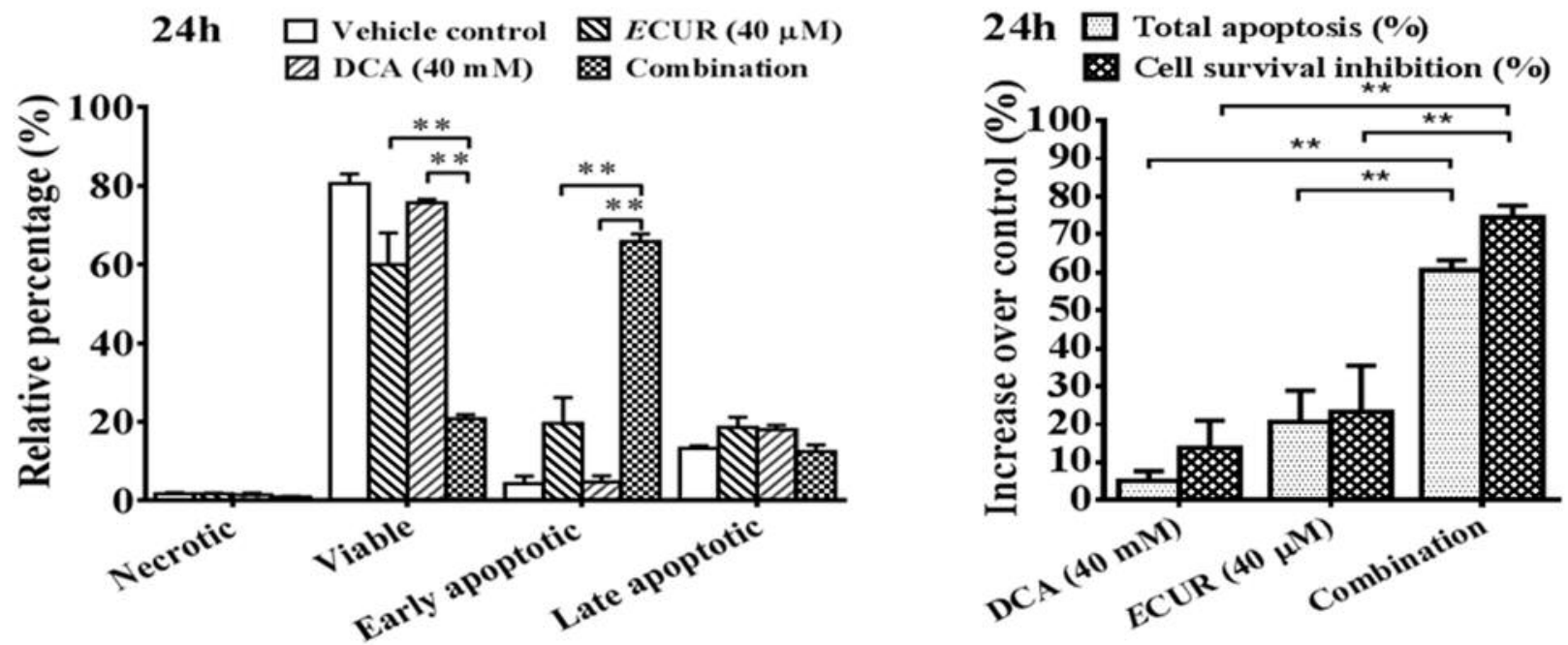

Figure 3. Continued 
D

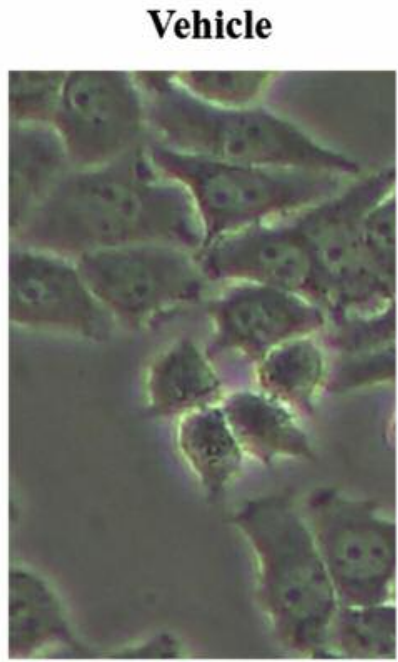

ECUR

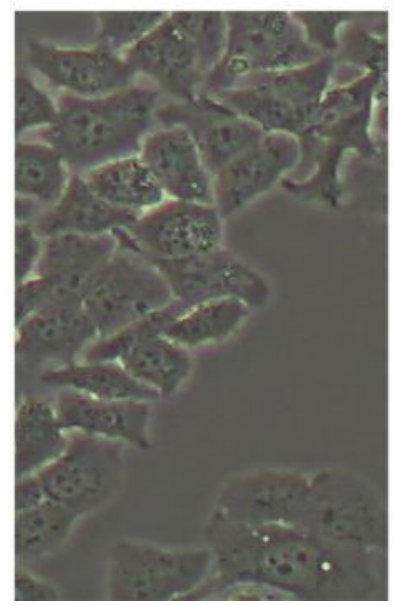

DCA

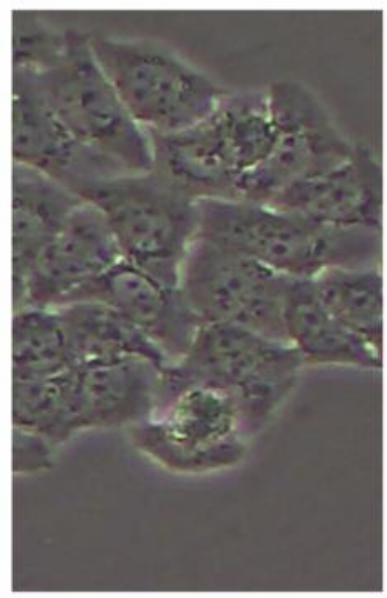

Combination

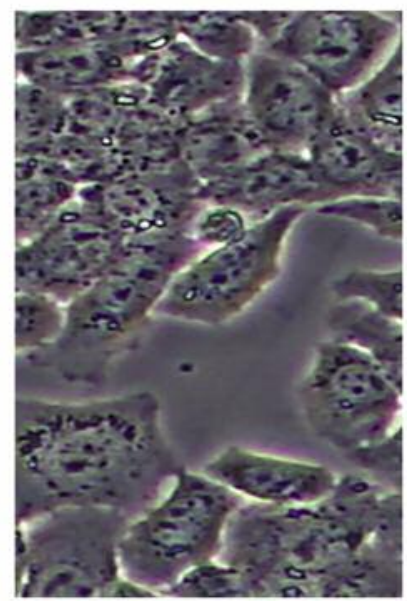

Figure 3. Quantitative apoptotic analysis and cellular morphological examination. The quantitative apoptotic analysis shows the relative percentages of necrotic, live, early apoptotic, and late apoptotic cells as measured by Muse ${ }^{T M}$ Annexin V and Dead Cell assay. Huh-7 cells were treated with vehicle, dichloroacetate (DCA), essential oil-blended curcumin (ECUR), or their combination for 16 and 24 h. A: The histogram is representative of four independent experiments. The quantitative graph shows that DCA treatment coupled with ECUR significantly enhanced cell apoptosis over both single treatments $(B)$; and the increase in the relative percentage of total apoptosis (early apoptosis plus late apoptosis) was consistent with the increase in the relative percentage of cell survival inhibition (derived from Figure $1 C$ ) (C). Data are expressed as means $\pm S D(N=4)$. ANOVA followed by Tukey's multiple comparisons test, significantly different at $* p<0.05$ and $* * p<0.01$. D: Apoptosis was further confirmed by cell morphological change using inverted microscopy (magnification, x200). Following treatment with DCA, ECUR and their combination for 24 hours, a proportion of Huh-7 cells exhibited nuclear fragmentation, indicating the occurrence of apoptotic processes.

treatment with curcumin may provide a feasible approach to simultaneously overcoming these two critical issues in cancer therapy, thereby enhancing therapeutic potential. Further studies are needed to test this critical hypothesis.

In summary, DCA synergized with ECUR and dramatically boosted the antiproliferative effect against hepatoma Huh-7 cells through enhancing apoptotic induction, probably by upregulating expression of pro-apoptotic BCL2 family proteins. Coupling DCA treatment with curcumin may rationally be expected to lower the DCA dose required and relatively reduce accompanying toxicity along with oxidative damage while enhancing the anticancer potential, thereby potentially opening a new therapeutic window. In this sense, this novel 'add-on' approach is of enormous value to current DCA therapy.

\section{Acknowledgements}

The Authors would like to thank Pelling Kan, who suffered from advanced lung adenocarcinoma, for her benevolence to inspire us to complete this anticancer study.

\section{References}

1 Mohammadian M, Mahdavifar N, Mohammadian-Hafshejani A and Salehiniya H: Liver cancer in the world: epidemiology, incidence, mortality and risk factors. World Cancer Res J 5(2): e1082, 2018.

\section{$24 \mathrm{~h}$}
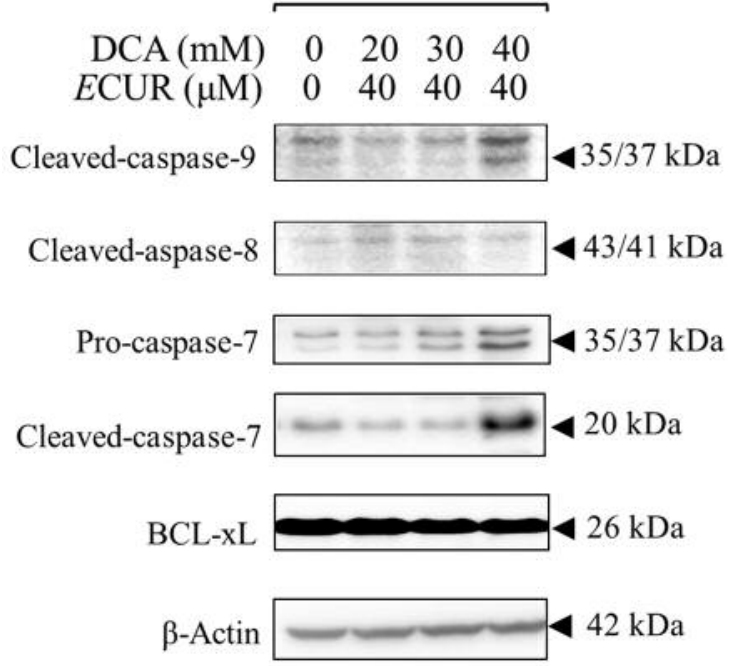

Figure 4. Combined effects of dichloroacetate (DCA) and essential oilblended curcumin (ECUR) on the expression of apoptosis-associated caspases and antiapoptotic B-cell lymphoma-extra large (BCL-xL) protein. Huh-7 cells were treated with increasing concentrations of DCA in combination with ECUR for $24 h$ and subjected to western blot analysis using antibodies against caspase-7, -8 and -9 and BCL-xL. $\beta$-Actin was used as a loading control. The combination treatments resulted in the increased expression of caspase-7 and -9 compared with the control, while the levels of cleaved caspase-8 and BCL-xL were not obviously affected. 

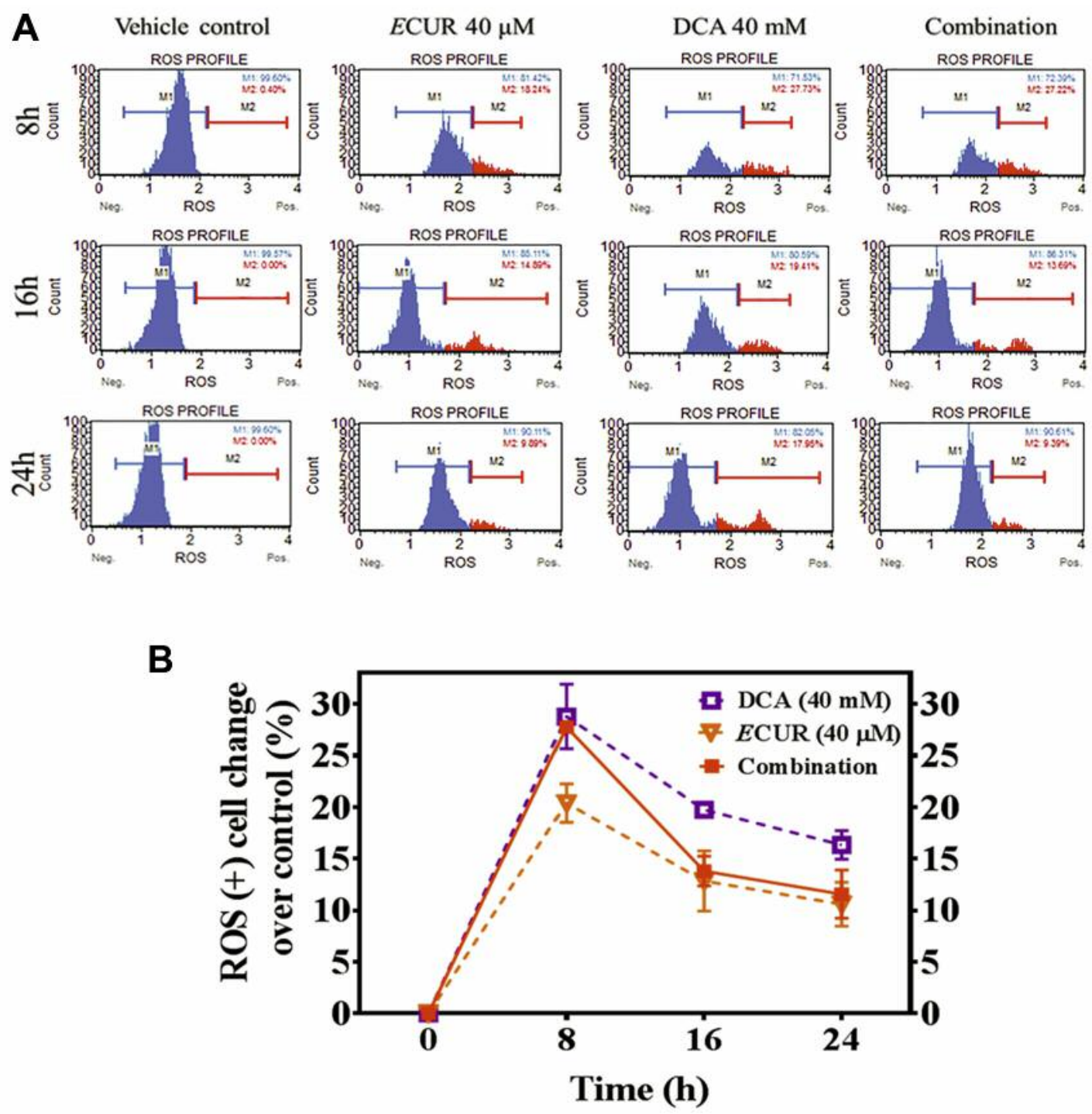

Figure 5. The quantitative intracellular reactive oxygen species (ROS) analysis. The quantitative ROS analysis shows the relative percentage of $\operatorname{ROS}^{-}(M 1)$ and $\operatorname{ROS}^{+}(M 2)$ cells resulting from Muse flow cytometry based oxidative stress assay ${ }^{T M}$. Huh-7 cells were treated with vehicle, dichloroacetate (DCA), essential oil-blended curcumin (ECUR), or their combination for 8,16 and $24 \mathrm{~h}$. A: The histogram is representative of three independent experiments. B: The quantitative graph shows that DCA and ECUR elevated the relative percentage of ROS ${ }^{+}(M 2)$ cells over the control. However, DCA treatment in combination with ECUR did not further promote production of that of ROS ${ }^{+}(M 2)$ cells over single treatment. Data are expressed as means $\pm S D(N=3)$.

2 Medavaram S and Zhang Y: Emerging therapies in advanced hepatocellular carcinoma. Exp Hematol Oncol 7: 17, 2018.

3 Bonnet S, Archer SL, Allalunis-Turner J, Haromy A, Beaulieu C, Thompson R, Lee CT, Lopaschuk GD, Puttagunta L, Bonnet S, Harry G, Hashimoto K, Porter CJ, Andrade MA, Thebaud B and Michelakis ED: A mitochondria-K+ channel axis is suppressed in cancer and its normalization promotes apoptosis and inhibits cancer growth. Cancer Cell 11: 37-51, 2007.

4 Dai Y, Xiong X, Huang G, Liu J, Sheng S, Wang H and Qin W: Dichloroacetate enhances adriamycin-induced hepatoma cell toxicity in vitro and in vivo by increasing reactive oxygen species levels. PLoS One 9(4): e92962, 2014.

5 Tran Q, Lee H, Park J, Kim SH and Park J: Targeting cancer metabolism-revisiting the Warburg effects. Toxicol Res 32(3): 177-193, 2016.

6 Khan A, Andrews D and Blackburn AC: Long-term stabilization of stage 4 colon cancer using sodium dichloroacetate therapy. World J Clin Cases 4(10): 336-343, 2016.

7 Aggarwal BB, Kumar A and Bharti AC: Anticancer potential of curcumin: preclinical and clinical studies. Anticancer Res 23: 363-398, 2003. 
8 Aggarwal S, Ichikawa H, Takada Y, Sandur SK, Shishodia S and Aggarwal BB: Curcumin (diferuloylmethane) down-regulates expression of cell proliferation and antiapoptotic and metastatic gene products through suppression of IKB $\alpha$ kinase and AKT activation. Mol Pharmacol 69(1): 195-206, 2006.

9 Antony B, Merina B, Iyer VS, Jud Antony B y N, Lennertz K and Joyal S: A pilot cross-over study to evaluate human oral bioavailability of BCM-95CG (Biocurcumax), a novel bioenhanced preparation of curcumin. Indian J Pharm Sci 70(4): 445-449, 2008.

10 Sun W and Cabrera R: Systemic treatment of patients with advanced, unresectable hepatocellular carcinoma: emergence of therapies. J Gastrointest Cancer 49(2): 107-115, 2018.

11 Cao SS and Zhen YS: Potentiation of antimetabolite antitumor activity in vivo by dipyridamole and amphotericin B. Cancer Chemother Pharmacol 24: 181-186, 1989.

12 Hao JQ, Li Q, Xu SP, Shen YX and Sun GY: Effect of lumiracoxib on proliferation and apoptosis of human nonsmall cell lung cancer cells in vitro. Chin Med J 121: 602-607, 2008.

13 Wang WZ, Cheng J, Luo J and Zhuang SM: Abrogation of $\mathrm{G}_{2} / \mathrm{M}$ arrest sensitizes curcumin-resistant hepatoma cells to apoptosis. FEBS Lett 582(18): 2689-2695, 2008.

14 Babu E, Ramachandran S, CoothanKandaswamy V, Elangovan S, Prasad PD, Ganapathy V and Thangaraju M: Role of SLC5A8, a plasma membrane transporter and a tumor suppressor, in the antitumor activity of dichloroacetate. Oncogene 30(38): 4026-4037, 2011.

15 Brandsma D, Dorlo TP, Haanen JH, Beijnen JH and Boogerd W: Severe encephalopathy and polyneuropathy induced by dichloroacetate. J Neurol 257(12): 2099-2100, 2010.

16 Gauthier A and Ho M. Role of sorafenib in the treatment of advanced hepatocellular carcinoma: An update. Hepatol Res 43(2): 147-154, 2013.

17 Brunelle JK and Letai A: Control of mitochondrial apoptosis by the BCL2 family. J Cell Sci 122(Pt 4): 437-441, 2009.
18 Guo XZ, Shao XD, Liu MP, Xu JH, Ren LN, Zhao JJ, Li HY and Wang D: Effect of bax, BCL2 and BCL-xL on regulating apoptosis in tissues of normal liver and hepatocellular carcinoma. World J Gastroenterol 8: 1059-1062, 2002.

19 Trachootham D, Alexandre J and Huang P: Targeting cancer cells by ROS-mediated mechanisms: a radical therapeutic approach? Nat Rev Drug Discov 8(7): 579-591, 2009.

20 Reuter S, Gupta SC, Chaturvedi MM and Aggarwal BB: Oxidative stress, inflammation, and cancer: How are they linked? Free Radic Biol Med 49(11): 1603-1616, 2010.

21 Hassoun EA, Cearfoss J and Spildener J: Dichloroacetate-and trichloroacetate-induced oxidative stress in the hepatic tissues of mice after long-term exposure. J Appl Toxicol 30(5): 450-456, 2010.

22 Li PM, Li YL, Liu B, Wang WJ, Wang YZ and Li Z: Curcumin inhibits MHCC97H liver cancer cells by activating ROS/TLR4/caspase signaling pathway. Asian Pac J Cancer Prev 15(5): 2329-2334, 2014.

23 Xia Y, Shen S and Verma IM: NF-kB, an active player in human cancers. Cancer Immunol Res 2(9): 823-830, 2014.

24 Schwartz L, Supuran CT and Alfarouk KO: The Warburg effect and the hallmarks of cancer. Anticancer Agents Med Chem 17(2): 164-170, 2017.

25 Strum SB, Adalsteinsson O, Black RR, Segal D, Peress NL and Waldenfels J: Case report: Sodium dichloroacetate (DCA) inhibition of the "Warburg Effect" in a human cancer patient: Complete response in non-Hodgkin's lymphoma after disease progression with rituximab-CHOP. J Bioenerg Biomembr 45(3): 307-315, 2013.
Received September 13, 2018

Revised October 14, 2018

Accepted October 16, 2018 